\title{
Admitting Spontaneous Violations of the Second Law in Continuum Thermomechanics
}

\author{
Martin Ostoja-Starzewski
}

Department of Mechanical Science \& Engineering, University of Illinois at Urbana-Champaign, Urbana, IL 61801-3620, USA; martinos@illinois.edu

Academic Editor: Daniel P. Sheehan

Received: 13 December 2016; Accepted: 16 February 2017; Published: 21 February 2017

\begin{abstract}
We survey new extensions of continuum mechanics incorporating spontaneous violations of the Second Law (SL), which involve the viscous flow and heat conduction. First, following an account of the Fluctuation Theorem (FT) of statistical mechanics that generalizes the SL, the irreversible entropy is shown to evolve as a submartingale. Next, a stochastic thermomechanics is formulated consistent with the FT, which, according to a revision of classical axioms of continuum mechanics, must be set up on random fields. This development leads to a reformulation of thermoviscous fluids and inelastic solids. These two unconventional constitutive behaviors may jointly occur in nano-poromechanics.
\end{abstract}

Keywords: continuum mechanics; fluctuation theorem; second law violations

\section{Introduction}

As is well known, the Second Law can be expressed in terms of a deterministic inequality (e.g., [1])

$$
\Delta S^{(i r)} \geq 0
$$

where $\Delta S^{(i r)}$ is the irreversible part of the entropy increment $\Delta S$. The later quantity can at any given time be written as the sum

$$
\Delta S=\Delta S^{(r)}+\Delta S^{(i r)} \text { with } \Delta S^{(r)}=\frac{\Delta Q}{\theta}
$$

in which $\Delta S^{(r)}$ is the reversible part of $S$, while $\theta$ is the absolute temperature.

While random fluctuations are negligible on macroscales, the Second Law gets spontaneously violated on very small (molecular) scales, as expressed by the so-called fluctuation theorem (FT) which gives the relative probability of observing processes that have positive $(A)$ and negative $(-A)$ total dissipation in non-equilibrium systems [2,3]:

$$
\frac{\mathrm{P}\left(\phi_{t}=A\right)}{\mathrm{P}\left(\phi_{t}=-A\right)}=e^{A t} .
$$

Here $\phi_{t}$ is the total dissipation for a trajectory $\Gamma \equiv\left\{q_{1}, p_{1}, \ldots, q_{N}, p_{N}\right\}$ of $N$ particles originating at $\Gamma(0)$ and evolving for a time $t$ :

$$
\phi_{t}(\boldsymbol{\Gamma}(0))=\int_{0}^{t} \phi(\Gamma(s)) d s .
$$

The integral in (3) involves an instantaneous dissipation function:

$$
\phi(\Gamma(t))=\frac{d \phi_{t}(\boldsymbol{\Gamma}(0))}{d t}
$$


It follows that the Second Law is correct as either an ensemble, or a temporal, or a volume average. Clearly, the FT represents a stochastic generalization of the Second Law. Depending on the particular assumptions/conditions, there exist similar versions of the FT [2]. Note that experiments validating the FT have been conducted $[4,5]$. The fluctuations are Gaussian.

There are three types of classical physics phenomena where spontaneous violations occur-viscous flow [6], heat conduction [7], and electrical resistance [3] —and in this paper we review the recently introduced extensions of continuum mechanics incorporating the first two of these. See also Figures 1 and 2 in [8] for molecular fluid dynamics simulations showing such violations.

\section{Irreversible Entropy Evolves as a Submartingale}

In view of the random fluctuations, $\phi_{t}$ is a stochastic process with a specific type of memory effect to be examined as follows. First, every stochastic process is defined with reference to a probability space $(\Omega, \mathcal{F}, \mathcal{P})$, where $\Omega$ is the sample space (of elementary events), $\mathcal{F}$ is the $\sigma$-field, and $\mathcal{P}$ the probability measure, the argument $\omega \in \Omega$ being employed to indicate an elementary event as well as the random character of $\phi_{t}$. We now switch from a continuous $(t)$ to a discrete $(n)$ time parametrization

$$
\phi_{n}:=\phi_{t=n}
$$

the reason for this switch being that the analytical aspects of discrete-time stochastic processes are simpler than those of continuous-time processes; the integral in (4) is replaced by a summation, while the derivative in (5) is understood in a finite-difference sense.

Our growing knowledge of the process $\phi_{n}$ at the successive times (i.e., its history) is represented by a so-called filtration on $\Omega$ : a sequence $\left\{\mathcal{F}_{n}: n=0,1,2, \ldots\right\}$ of sub-sigma fields of $\mathcal{F}$ such that for all time instants $t_{n}, \mathcal{F}_{n} \subset \mathcal{F}_{n+1}$. In view of (5), we observe that this inequality is satisfied

$$
\mathrm{E}\left\{\phi_{n+1} \mid \mathcal{F}_{n}\right\} \geq \phi_{n}
$$

which indicates that $\phi_{n}$ is a submartingale. On the technical side dictated by the probability theory, (7) has to be accompanied by two more conditions: (i) $\left\{\mathcal{F}_{n} ; n=0,1,2, \ldots\right\}$ is a filtration and $\phi_{n}$ is adapted to $\mathcal{F}_{n}$; (ii) for each $n, \phi_{n}$ is integrable.

If the $\geq$ sign in (7) were replaced by an equality sign, we would have a so-called martingale. In fact, this observation acquires more light in view of the so-called Doob decomposition [9] saying that any submartingale is the sum of a martingale $(M)$ and an increasing process $(G)$ : Let $\phi=\left\{\phi_{n} ; n \geq 0\right\}$ be a submartingale relative to the filtration $\left(\mathcal{F}_{n}\right)$. Then there exists a martingale $M=\left\{M_{n} ; n \geq 0\right\}$ and a process $G=\left\{G_{n} ; n \geq 0\right\}$ such that

(i) $M$ is a martingale relative to $\mathcal{F}_{n}$;

(ii) $G$ is an increasing process: $G_{n} \leq G_{n+1}$ almost everywhere;

(iii) $G_{n}$ is $\mathcal{F}_{n-1}$-measurable $\forall n$;

(iv) $\phi_{n}=M_{n}+G_{n}$.

In [10] we have employed an analogous (Doob-Meyer decomposition) theorem in continuous time, also giving a unique decomposition of a submartingale into a martingale and a "drift" process. The discrete time case should be sufficient for most continuum physics applications, while allowing a simpler analytical treatment.

Since we are interested in continuum mechanics/physics, we write Equations (1) and (2) in terms of the rates of entropy densities $\left(\dot{s}, s^{*(r)}, s^{*(i r)}\right)$

$$
\dot{s}=s^{*(r)}+s^{*(i r)} \text { with } s^{*(r)}=-\left(\frac{q_{i}}{\theta}\right), i \text { and } s^{*(i r)} \geq 0,
$$

where $q_{i}(\equiv \mathbf{q})$ is the heat flux. As we are focusing on continuum physics, we use equivalent notations of subscript-type $\left(f_{i \ldots}\right)$ and symbolic-type (f) for tensors. While an overdot indicates a material time 
derivative corresponding to a total differential of a given quantity, a superscript * is used to indicate a time rate of a quantity which is not a state function $[1,11]$.

While the inequality in $(8)_{3}$ is assumed to hold instantaneously (i.e., for $\forall t$ ) in conventional continuum mechanics/physics, in fact it holds only for statistical averages (such as (7) above), or for sufficiently long time averages, or for sufficiently large systems. We are interested in extending the conventional continuum mechanics/physics so as to account for violations of the Second Law according to FT.

\section{Stochastic Thermomechanics}

\subsection{Basic Framework}

Since the fluctuation theorem involves a dissipation function, the natural framework to generalize the continuum mechanics is one employing the thermodynamics with internal variables (TIV) [1], where the key role is played by the internal energy $u$ and the entropy production rate $\dot{s}^{(i)}$. Thus, $u=u\left(\varepsilon_{i j}, s\right)$ is the functional of strain $\varepsilon_{i j}$ (assuming for simplicity that we work with small elastic strains) and entropy $s$, satisfying the first law of thermodynamics (the energy balance)

$$
\rho \dot{u}=\sigma_{i j} d_{i j}-q_{i, i},
$$

where $\rho$ is the mass density, $\sigma_{i j}$ is the Cauchy stress, $d_{i j}$ is the deformation rate, and $q_{i}$ is the heat flux. Note that the first term on the right is the specific power of deformation. By a well-known partial Legendre transform, the free energy is

$$
\psi\left(\varepsilon_{i j}, \theta\right)=u\left(s, \varepsilon_{i j}\right)-s \theta .
$$

Following the standard derivations of TIV [11], on account of $(8)_{1},(8)_{2},(9)$, and (10), we obtain the relation

$$
\sigma_{i j}^{(d)} d_{i j}=\rho \frac{\partial \psi}{\partial \varepsilon_{i j}} d_{i j}+\frac{q_{i}}{\theta} \theta_{, k}+\rho \theta \dot{s}^{(i r)}
$$

With $\psi$ playing the role of a potential for the entropy $(s)$ and the quasi-conservative Cauchy stress $\left(\sigma_{i j}^{(q)}\right)$, we have

$$
s=-\frac{\partial \psi}{\partial \theta}, \quad \sigma_{i j}^{(q)}=\frac{\partial \psi}{\partial \varepsilon_{i j}} .
$$

The stress $\sigma_{i j}^{(d)}$ is the dissipative part of the total stress $\sigma_{i j}\left(=\sigma_{i j}^{(q)}+\sigma_{i j}^{(d)}\right)$; it appears in the discussion of irreversible phenomena immediately below.

If we were to assume $(8)_{3}$, we would obtain the standard Clausius-Duhem inequality

$$
\mathbf{Y} \cdot \mathbf{V}=\sigma_{i j}^{(d)} d_{i j}-q_{k} \frac{\theta, k}{\theta}=\rho \theta \dot{s}^{(i r)} \geq 0,
$$

where we employ a standard representation of $\phi$ as a scalar product of two conjugate vectors

$$
\begin{array}{cl}
\text { velocities } & \mathbf{V}=\left\{d_{i j}, \theta_{r k} / \theta\right\}, \\
\text { dissipative forces } & \mathbf{Y}=\left\{\sigma_{i j}^{(d)},-q_{k}\right\} .
\end{array}
$$

If we admit violations of the Second Law according to the FT discussed in the first section of this paper, the dissipation function $\phi=\theta \dot{s}^{(i)}$ is neither strictly non-negative for all times, nor deterministic. Rather, $\phi$ is a scale-dependent stochastic functional $\phi(\mathbf{V}, \omega)$, which satisfies the Clausius-Duhem inequality only in the ensemble average sense

$$
\mathrm{E}\{\mathbf{Y} \cdot \mathbf{V}\}=\mathrm{E}\{\phi(\mathbf{V}, \omega)\} \geq 0 .
$$


The explicit dependence of $\phi$ on $\omega(\in \Omega)$ indicates the stochastic character of $\phi$. The basic model, sufficiently general for our purposes and valid for linear viscous (Stokes-type) and thermal (Fourier-type) fluids, involves an additive decomposition of $\phi$ into two parts: one mechanical $\left(\phi_{\text {mech }}\right)$ and another thermal $\left(\phi_{t h}\right)$ :

$$
\phi(\mathbf{V}, \omega)=\phi_{m e c h}\left(d_{i j}, \omega\right)+\phi_{t h}\left(\theta_{, k} / \theta, \omega\right) .
$$

Now, the randomness itself is introduced by taking each of these parts as a sum of a deterministic mean (which must be positive-valued) plus a fluctuating part which may spontaneously take negative values, see (19) below.

\subsection{Axioms of Continuum Thermomechanics}

In view of the preceding discussion, the fundamental rôle in TIV [1] is played by the free energy $(\psi)$ and the dissipation function $(\phi)$, the latter quantity, upon (temporal, volume, or statistical) averaging, giving the positive entropy production rate. Clearly, TIV lends itself to an extension replacing the Second Law by the FT. Notably, an amazingly wide range of continuum constitutive behaviors can be obtained from the pair $\{\psi, \phi\}[1,11,12]$.

By contrast, in the rational continuum mechanics (RCM) one works with a quartet of the stress state $\sigma_{i j}$, heat flux $q_{i}$, free energy density $\psi$, and entropy $s$, all of which are taken as functionals of the history at a continuum point. As outlined in [13], the axioms (also called principles) of RCM need to be modified so as to admit a negative entropy production:

1. The Axiom of Causality: "The future state of the system depends solely on the probabilities of events in the past". That is, quoting Evans \& Searles [3]: "the probability of subsequent events can be predicted from the probabilities of finding initial phases and a knowledge of preceding changes in the applied field and environment of the system."

2. The FT is derived from the Axiom of Causality. While the entropy $s^{(i)}$ is a submartingale in time $(\mathcal{T}=(-\infty, t])$, it is a random field in spatial domain $\mathcal{D}$ :

$$
\phi: \mathcal{T} \times \mathcal{D} \times \Omega \rightarrow \mathbb{R}
$$

3. The Second Law in its conventional deterministic form is obtained-upon spatial, statistical, or time averaging-as a special case of the fluctuation theorem, and the entire field of continuum mechanics is recovered as a special case.

4. The Axiom of Determinism: "at any instant $t$, the value of $\left[\sigma_{i j}, q_{i}, u, s\right]$ at depends on the whole history of the thermokinetic process up to the time $t$ ". The choice of the thermokinetic process depends on the particular physics involved, and may take the form of a classical or non-classical (e.g., micropolar) theory or a stochastic field.

5. The Axiom of Local Action is to be replaced by the scale dependence of the adopted continuum approximation for, clearly, that axiom makes no clear reference to the microstructure of the medium.

6. The Axiom of Equipresence (all the constitutive quantities depend a priori on the same variables) is to be abandoned since the violation of Second Law may occur in one physical process present in constitutive relations, not all.

\subsection{Random Fields}

In view of the preceding discussion, the deterministic continuum theory must be replaced by a stochastic one: working with TIV, the free energy density $\psi$ and the dissipation function $\phi$ are real-valued random fields over the material $(\mathcal{D})$ and time $(\mathcal{T}=(-\infty, t])$ domains:

$$
\psi: \mathcal{D} \times \mathcal{T} \times \Omega \rightarrow \mathbb{R}, \quad \phi: \mathcal{D} \times \mathcal{T} \times \Omega \rightarrow \mathbb{R},
$$


For example, considering the thermal dissipation, we have

$$
\phi_{t h}\left(\frac{\nabla \theta}{\theta}, \omega\right)=-\mathbf{q} \cdot \frac{\nabla \theta}{\theta} \equiv-q_{k} \frac{\theta, k}{\theta} .
$$

Given the spontaneous, randomly occurring violations of the Second Law, the dissipation function reads

$$
\phi_{t h}\left(\frac{\theta_{r k}}{\theta}, \omega\right)=\dot{G}\left(\frac{\theta_{r k}}{\theta}\right)+\dot{M}\left(\frac{\theta_{r k}}{\theta}, \omega\right) .
$$

In the case of linear Fourier-type conductivity, (20) becomes more explicit with

$$
\dot{G}\left(\frac{\theta_{r k}}{\theta}\right)=\frac{1}{\theta} \theta_{r i} k_{i j} \theta_{, j}, \quad \dot{M}\left(\frac{\theta_{, k}}{\theta}, \omega\right)=\frac{1}{\theta} \theta_{r i} \mathcal{M}_{i j}(\omega) \theta_{, j} .
$$

Here $\dot{G}\left(\theta_{, k} / \theta\right)$ involves the deterministic thermal conduction $k_{i j}$ (which is positive definite) and $\dot{M}\left(\theta_{, k} / \theta, \omega\right)=d M(\mathbf{q}, \omega) / d t$, with $M$ being the martingale modeling the random fluctuations according to the Doob decomposition (iv) in Section 2. Clearly, the randomness residing in $\dot{M}$ allows the total thermal conductivity $k_{i j}+\mathcal{M}_{i j}$ to sometimes become negative-definite, since $\mathcal{M}_{i j}$ is not required to be positive-definite, thus signifying the violations of the Second Law. More specifically, $\mathcal{M}_{i j}: \mathcal{V} \rightarrow \mathcal{V}$ (where $\mathcal{V}$ is a real vector space) is a second-order, rank 2 tensor random field (e.g., [14,15])

$$
\mathcal{M}_{i j}: \mathcal{D} \times \Omega \rightarrow \mathcal{V}^{2}
$$

In view of the Gaussian character of nanoscale fluctuations (recall Section 1 ), $\mathcal{M}_{i j}$ is a Gaussian tensor random field, making negative values possible.

By analogy, the same type of approach may be used to introduce fluctuations in mechanical dissipation $\phi_{\text {mech }}\left(d_{i j}, \omega\right)$ having spontaneous violations of the Second Law, recall (15).

\section{Thermoviscous Fluids}

We start from the free energy and the dissipation function

$$
\psi=\psi\left(\varepsilon_{i j}, \theta, \dot{\theta}\right) \text { and } \phi=\phi\left(d_{i j}, \vartheta, i / \theta\right),
$$

and assume the macroscopic incompressibility

$$
\varepsilon_{i i}=0 \quad \text { and } \quad d_{i i}=0 .
$$

The free energy function $\psi$ (taken per unit volume), assuming no elastic response but the presence of a relaxation time $t_{0}$, is

$$
\psi\left(\varepsilon_{i j}, \theta, \dot{\theta}\right)=\psi_{0}-s_{0} \vartheta-\frac{C_{E}}{2 \theta_{0}} \vartheta^{2}-\frac{C_{E}}{\theta_{0}} t_{0} \vartheta \dot{\vartheta}, \quad \vartheta:=\theta-\theta_{0} .
$$

Here $\psi_{0}$ and $s_{0}$ are the free energy and entropy in the reference state, $\mu$ is the shear elastic modulus, $C_{E}$ is the specific heat at constant strain, and $\vartheta$ is the temperature difference from the reference temperature $\theta_{0}$. The last term on the right hand side is taken by analogy to the thermoelasticity with two relaxation times in the next section, so as to retain the Fourier-type heat conduction, but to obey the hyperbolic (finite speed) heat propagation. In this section, an overdot denotes a material derivative for absolute tensors (like the temperature gradient and deformation rate) and an Oldroyd derivative for tensor densities (like the heat flux and stress tensor). 
The free energy being a potential for quasi-conservative stresses $\sigma_{i j}^{(q)}$ and the entropy s, we find

$$
\begin{gathered}
\sigma_{i j}^{(q)}=-p \delta_{i j}, \\
s=-\frac{\partial \psi}{\partial \theta}=\frac{C_{E}}{\theta_{0}} \vartheta+\frac{C_{E}}{\theta_{0}} t_{0} \dot{\vartheta} .
\end{gathered}
$$

The first relation above reflects the presence of a hydrostatic pressure and the fact that $\psi$ does not depend on the strain $\varepsilon_{i j}$ since the fluid under consideration does not have any elasticity. The relation $(26)_{2}$ is immediately identified as the constitutive equation for entropy.

Henceforth, in view of the fluid's incompressibility in $(24)_{1}$ and to simplify the notation, $\sigma_{i j}^{(q)}=0$ is taken as the deviatoric part of the quasi-conservative stress tensor. As always in TIV [1], there holds the relation

$$
\sigma_{i j}=\sigma_{i j}^{(q)}+\sigma_{i j}^{(d)}
$$

where $\sigma_{i j}^{(d)}$ is the dissipative stress, which now also represents the deviatoric part only.

This Ansatz leads to the Clausius-Duhem inequality in the form

$$
\rho \theta s^{*(i r)}=-\frac{q_{i} \vartheta_{, i}}{\theta}+\sigma_{i j}^{(d)} d_{i j} \geq 0
$$

where $\sigma_{i j}^{(d)}$ is the dissipative stress, which is now equal to the total stress $\sigma_{i j}$. As discussed earlier, the inequality (28) may spontaneously be violated according to the FT.

On account of the equality in (28), we take the specific (per unit volume) dissipation $\phi$ as a functional of temperature gradient $\vartheta_{, i} / \theta$ and deformation rate $d_{i j}$ :

$$
\rho \theta s^{*(i r)}=\phi\left(d_{i j},-\vartheta, i / \theta\right) .
$$

Therefore, the inequality in (28) may be stated in terms of the scalar product: $\mathbf{Y} \cdot \mathbf{V} \geq 0$.

Next, for the entropy production rate we adopt the dissipation functional in the space of velocity $\mathbf{V}=(\mathbf{d},-\nabla \theta / \theta)$ :

$$
\rho \theta s^{*(i r)} \equiv \phi(\mathbf{V})=H d_{i j} d_{i j}+\frac{k}{\theta} \vartheta_{, i} \vartheta_{, i}
$$

where $k$ is the Fourier conductivity and $H$ the fluid viscosity, both parameters modeling an isotropic medium. The Equation (30) is a special case of (29), with both processes being effectively compound [1]. Using the thermodynamic orthogonality, (30) yields

$$
\begin{gathered}
\sigma_{i j}=\sigma_{i j}^{(d)}=\frac{1}{2} \frac{\partial \phi}{\partial d_{i j}}=H d_{i j} \\
-q_{i}=\frac{1}{2} \frac{\partial \phi}{\partial \vartheta_{, i}}=k \vartheta_{, i} .
\end{gathered}
$$

Collecting the three parts of the constitutive law-mechanical (Stokesian), thermal (Fourier law), and entropy- and introducing the FT-type fluctuations, we have

$$
\begin{gathered}
\sigma_{i j}=(H+\mathcal{H}) d_{i j}, \\
q_{i}=-(k+\mathcal{M}) \vartheta_{, i}, \\
s=\frac{C_{E}}{\theta_{0}}\left(\vartheta+t_{0} \dot{\vartheta}\right) .
\end{gathered}
$$


Here, on account of Section 3, we have introduced the martingale-type fluctuation $\mathcal{M}$ in thermal response $(32)_{2}$ and an analogous one $(\mathcal{H})$ in the viscous response $(32)_{1}$. Relation $(32)_{3}$ shows that, while the Fourier-type law holds, there is a relaxation effect involved in the entropy. If the scale of the elementary volume $d V$ of continuum mechanics approximation is very small, that element will see violations of the Second Law according to [7]. In this model the heat is conducted with finite speeds-i.e., not infinite speeds as would be the case with $t_{0}=0$. In other words, instead of having a parabolic (diffusion) equation for temperature, we have (by application of the energy balance (24) and the entropy-temperature relation $\left.(32)_{3}\right)$

$$
k \vartheta, i i=\rho C_{E}\left(\dot{\vartheta}+t_{0} \ddot{\vartheta}\right) .
$$

Here we have also used the approximation of small temperature fluctuations. In effect, $\vartheta$ (and also $\theta$ ) is governed by the telegraph (damped hyperbolic) Equation (33), whose limiting case (for $t_{0} \rightarrow 0$ ) is the conventional (parabolic) heat conduction equation.

\section{Inelastic Solids}

\subsection{Acceleration Wave with Nanoscale Wavefront Thickness}

An acceleration wave in continuum mechanics is a surface carrying a jump $(\alpha)$ in particle acceleration. In a very wide class of nonlinear elastic/dissipative media the amplitude of acceleration wave is governed by a Bernoulli equation (e.g., $[16,17])$

$$
\frac{d \alpha}{d t}=-\mu \alpha+\beta \alpha^{2}
$$

Here the coefficients $\mu$ and $\beta$ represent, respectively, two effects: dissipation and elastic nonlinearity. Clearly, there is a competition between these two effects as the wave propagates, and there exists a possibility of blow-up $(\alpha \rightarrow \infty)$, and hence, of shock formation in a finite time $t_{\infty}$, providing the initial amplitude $\alpha_{0}$ exceeds a critical amplitude $\alpha_{c}$

$$
\alpha_{c}=\frac{\mu}{\beta} \quad t_{\infty}=-\frac{1}{\mu} \ln \left(1-\frac{\mu}{\beta \alpha_{0}}\right) .
$$

When the analysis is conducted in the spatial domain (as done below), a shock (or caustic) forms at $x_{\infty}$ called the distance to blow-up or distance to form a shock.

If the acceleration wavefront is truly of nanoscale thickness, then the dissipation is not only fluctuating randomly as seen by an observer traveling with the wavefront, but may also take negative values according to the fluctuation theorem. At this point it is important to note the formulas specifying both material parameters in (34) as well as the wave speed $c$ :

$$
\mu=-\frac{G_{0}^{\prime}}{2 G_{0}}, \quad \beta=-\frac{\widetilde{E}_{0}}{2 G_{0} c}, \quad \text { with } \quad c=\sqrt{\frac{G_{0}}{\rho_{R}}},
$$

where $G_{0}(>0)$ is called the instantaneous modulus, $G_{0}^{\prime}$ (conventionally $\geq 0$ ) is the viscosity coefficient responsible for dissipation, $\widetilde{E}_{0}(\leq 0)$ is called the instantaneous second-order tangent modulus, and $\rho_{R}$ is the mass density in the reference state. According to FT, in (36) we admit $G_{0}^{\prime}$ to be randomly taking negative values while being positive on average. Analyzing such a stochastic dynamical system [8], we found: (i) the blow-up event becomes impossible as the wavefront thickness is larger because then, according to Equation (3), the probability of negative viscosity goes to zero; (ii) taking the spatial correlations of the random field viscosity richer than the white-noise (e.g., Ornstein-Uhlenbeck) does not fundamentally change the basic results reported here; (iii) as $\zeta_{0}$ gets larger, the probability of blow-up decreases to zero. 


\subsection{Thermoelasticity with Two Relaxation Times}

The classical thermo-elastodynamics is governed by a system of partial differential equations that are hyperbolic in elastic response and parabolic in heat conduction. While there are several way to replace the Fourier law by a more general heat conduction equation, so far the fluctuation theorem has been developed for a Fourier-type law only. Therefore, one may proceed with a theory of thermoelasticity with two relaxation times which is hyperbolic in elastic and thermal responses and, actually, involves the Fourier law [18]. To that end, we adopt the free energy (with $\vartheta=\theta-\theta_{0}$ as before)

$$
\psi=\psi\left(\varepsilon_{i j}, \theta\right)=\frac{1}{2} \varepsilon_{i j} C_{i j k l} \varepsilon_{k l}+M_{i j} \varepsilon_{i j} \vartheta-\frac{C_{E}}{2 \theta_{0}} \vartheta^{2}-\frac{C_{E}}{\theta_{0}} t_{0} \vartheta \dot{\vartheta}
$$

so that

$$
\sigma_{i j}^{(q)}=C_{i j k l} \varepsilon_{k l}+M_{i j} \vartheta \quad \text { and } s=-M_{i j} \varepsilon_{i j}+\frac{C_{E}}{\theta_{0}} \vartheta+\frac{C_{E}}{\theta_{0}} t_{0} \dot{\vartheta} .
$$

Here $C_{i j k l}, M_{i j}$ and $C_{E}$ denote the stiffness tensor, the stress-temperature tensor, and the specific heat at zero strain, respectively. These quantities satisfy the following symmetry and positive-definiteness relations

$$
\begin{gathered}
C_{i j k l}=C_{j i k l}=C_{i j l k}=C_{k l i j}, \quad \varepsilon_{i j} C_{i j k l} \varepsilon_{k l}>0, \\
M_{i j}=M_{j i}, \quad C_{E}>0 .
\end{gathered}
$$

Also, we adopt the dissipation function (this time in the space of heat flux and strain rate)

$$
\phi\left(q_{i}, \dot{\varepsilon}_{i j}\right)=T s^{*(i r)}=\frac{\lambda_{i j}}{\theta} q_{i} q_{j}+t_{1} M_{i j} \dot{\varepsilon}_{i j} \dot{\vartheta},
$$

where $\lambda_{i j}$ is the thermal resistivity, so that, by treating both processes as compound [1], we obtain

$$
-\frac{\vartheta_{, i}}{\theta}=\frac{1}{2} \frac{\partial \phi}{\partial q_{i}}=\frac{\lambda_{i j}}{\theta} q_{j} \quad \text { and } \quad \sigma_{i j}^{(d)}=\frac{\partial \phi}{\partial \dot{\varepsilon}_{i j}}=t_{1} M_{i j} \dot{\vartheta}
$$

With $\lambda_{i j}=\left(k_{i j}+\mathcal{M}_{i j}\right)^{-1}$, this leads to

$$
\begin{gathered}
\sigma_{i j}=\sigma_{i j}^{(q)}+\sigma_{i j}^{(d)}=C_{i j k l} \varepsilon_{k l}+M_{i j}\left(\vartheta+t_{1} \dot{\vartheta}\right), \\
\theta_{0} s=-\theta_{0} M_{i j} \varepsilon_{i j}+C_{E}\left(\vartheta+t_{0} \dot{\vartheta}\right), \\
q_{i}=-\left(k_{i j}+\mathcal{M}_{i j}\right) \vartheta_{, j},
\end{gathered}
$$

where, again (recalling Section 2), $\mathcal{M}_{i j}$ is a random field in space-time with spontaneous violations of positive-definiteness property. $\mathcal{M}_{i j}$ is not to be confused with $M_{i j}$, which is responsible for a thermal expansion-type of effect. Note that the total Fourier-type conductivity is $\left(k_{i j}+\mathcal{M}_{i j}\right)$ per $(42)_{3}$, i.e., the anisotropy being possible because we are now dealing with a solid, not a fluid as in Section 4.

It is well known [18] that, mathematically, the relations (42) lead to coupled and hyperbolic-type equations for the $\left(u_{i}, \vartheta\right)$ pair

$$
\begin{gathered}
\left(C_{i j k l} u_{k, l}\right)_{, j}-\rho \ddot{u}_{i}+\left[M_{i j}\left(\vartheta+t_{1} \dot{\vartheta}\right)\right]_{, j}=-b_{i}, \\
\left(\left(k_{i j}+\mathcal{M}_{i j}\right) \vartheta_{, j}\right)_{, i}-C_{E}\left(\dot{\vartheta}+t_{0} \ddot{\vartheta}\right)+\theta_{0} M_{i j} \dot{u}_{i, j}=-r,
\end{gathered}
$$


where $b_{i}$ and $r$ are the body force and heat source fields, respectively.

We observe:

(i) The constitutive relations (42) are the same as those of the Green-Lindsay theory, but their derivation is based on treating the Fourier-type heat conduction as a purely dissipative process, and thus as a process described by the dissipation function rather than by the free energy function only.

(ii) The inequalities $t_{1} \geq t_{0} \geq 0$ have to hold. By setting $t_{1}=t_{0}=0$, we obtain the classical thermoelasticity. Also, one may only consider the limit $t_{0} \rightarrow 0$, so that $(43)_{2}$ reduces to the conventional heat conduction equation.

(iii) Transient phenomena, if occurring on very short length scales (such as wavefronts discussed in the preceding section), are expected to deviate from the hyperbolic thermo-elastodynamics obeying the Second Law.

\section{Closure}

The situations where violations of the Second Law are relevant involve very small length scales (extremely thin wavefronts, nano-channels, nano-rods, ...) and short time scales (although times up to $3 \mathrm{~s}$ have been observed for cholesteric liquids [3]). Thus, a natural setting in which to expect such violations is that of poromechanics involving nanoscale channels, where the Second Law may apply only on average in the fluid within the channels (viscous flow and heat conduction) and the solid skeleton (heat conduction). The third possible phenomenon (electrical resistance) is one more possibility, that still needs to be worked out. As a reference, we start from the classical poromechanics obeying the Second Law [19], the Clausius-Duhem inequality is written in terms of the irreversible entropy production $S^{*(i r)}\left(=\rho s^{*(i r)}\right)$ taking the form

$$
T S^{*(i r)}=T S_{(\text {fluid })}^{*(i r)}+T S_{(\text {th })}^{*(i r)}+T S_{(\text {matrix })}^{*(\text { ir })} \geq 0,
$$

where three possible contributions to dissipation are identified: (1) fluid dissipation: $S_{(\text {fluid })}^{*(\text { ir })}$; (2) thermal dissipation: $S_{(t h)}^{*(i r)}$; (3) skeleton dissipation: $S_{(\text {matrix })}^{*(i r)}$. Conventionally, each of these contributions to dissipation is assumed to satisfy its own second law inequality. It now follows that, in the case of poromechanics describing phenomena on very small space and time scales, the spontaneous violations of the second law (43) can occur in either one, two or three processes.

Conflicts of Interest: The author declares no conflict of interest.

\section{References}

1. Ziegler, H. An Introduction to Thermomechanics; North Holland Publishing Co.: Amsterdam, The Netherlands, 1983.

2. Evans, D.J.; Cohen, E.G.D.; Morriss, G.P. Probability of second law violations in steady states. Phys. Rev. Lett. 1993, 71, 2401.

3. Evans, D.J.; Searles, D.J. The fluctuation theorem. Adv. Phys. 2002, 51, 1529-1585.

4. Ciliberto, C.; Laroche, C. An experimental test of the Gallovotti-Cohen fluctuation theorem. J. Phys. IV Fr. 1998, 8, Pr6-215-Pr6-215219.

5. Wang, G.M.; Sevick, E.M.; Mittag, E.; Searles, D.J.; Evans, D.J. Experimental demonstration of violations of the second law of thermodynamics for small systems and short time scales. Phys. Rev. Lett. 2002, 89, 050601.

6. Evans, D.J.; Morriss, G.P. Statistical Mechanics of Nonequilibrium Liquids, 2nd ed.; Cambridge University Press: Cambridge, UK, 2014.

7. Searles, D.J.; Evans, D.J. Fluctuation theorem for heat flow. Int. J. Thermophys. 2001, 22, 123-134.

8. Ostoja-Starzewski, M.; Raghavan, B. Continuum mechanics versus violations of the second law of thermodynamics. J. Therm. Stress. 2016, 39, 734-749.

9. Doob, J.L. Stochastic Processes; John Wiley \& Sons: Hoboken, NJ, USA, 1953. 
10. Ostoja-Starzewski, M.; Malyarenko, A. Continuum mechanics beyond the second law of thermodynamics. Proc. R. Soc. A 2014, 470, 20140531.

11. Ziegler, H.; Wehrli, C. The derivation of constitutive relations from the free energy and the dissipation functions. Adv. Appl. Mech. 1987, 25, 183-238.

12. Maugin, G.A. The Thermomechanics of Nonlinear Irreversible Behaviors-An Introduction; World Scientific: Singapore, 1999.

13. Ostoja-Starzewski, M. Second law violations, continuum mechanics, and permeability. Contin. Mech. Thermodyn. 2016, 28, 489-501; Erratum in 2017, 29, 359.

14. Malyarenko, A.; Ostoja-Starzewski, M. Statistically isotropic tensor random fields: Correlation structures. Math. Mech. Complex Syst. 2014, 2, 209-231.

15. Malyarenko, A.; Ostoja-Starzewski, M. Spectral expansions of homogeneous and isotropic tensor-valued random fields. Z. Angew. Math. Phys. 2016, 67, 1-20.

16. Bland, D.R. Nonlinear Dynamic Elasticity; Blaisdell: Waltham, MA, USA, 1969.

17. Chen, P.J. Selected Topics in Wave Propagation; Noordhoff International: Franklin Park, IL, USA, 1976.

18. Ignaczak, I.; Ostoja-Starzewski, M. Thermoelasticity with Finite Wave Speeds; Oxford University Press: Oxford, UK, 2010.

19. Coussy, O. Poromechanics; John Wiley \& Sons: Hoboken, NJ, USA, 2004.

(C) 2017 by the author. Licensee MDPI, Basel, Switzerland. This article is an open access article distributed under the terms and conditions of the Creative Commons Attribution (CC BY) license (http:/ / creativecommons.org/licenses/by/4.0/). 